\title{
Our experience with single patch repair of complete atrioventricular septal defects
}

\section{Can Vuran ${ }^{1 *}$, Uygar Yoruker ${ }^{2}$, Oguz Omay ${ }^{3}$, Bulent Saritas ${ }^{4}$, Canan Ayabakan ${ }^{5}$, Ozlem Sarisoy ${ }^{6}$ and Riza Turkoz ${ }^{7}$}

1Department of Pediatric Cardiovascular Surgery, University of Health Sciences Istanbul Umraniye, Training and research Hospital, Elmalıkent, Adem Yavuz Cd.No:1, 34764 Ümraniye, Istanbul,

Turkey

${ }^{2}$ Deparments of Pediatric Cardiac Surgery, Justus-Liebig-Universität Gießen, Germany

${ }^{3}$ Department of Cardiovascular Surgery, Kocaeli University, Kocaeli, Turkey

${ }^{4}$ Department of Cardiovascular Surgery, Aydin University, Istanbul, Turkey

${ }^{5}$ Department of Pediatric Cardiology, Baskent University, Istanbul, Training and Research Hospital, Istanbul, Turkey

${ }^{6}$ Department of Pediatric Cardiology, University of Health Sciences, Istanbul, Umraniye Training and Research Hospital, Istanbul, Turkey

${ }^{7}$ Department of Cardiovascular Surgery, Acibadem Healthcare Services, Istanbul, Turkey

\section{Abstract}

Background: Various surgical methods have been utilized in the management of complete atrioventricular septal defects (CAVSD). Early intervention and achievement of a competent left atrioventricular valve are the key factors for successful treatment.

Methods: A total of 66 patients with complete atrioventricular septal defect have been operated in a tertiary care center. Patient group consisted of 28 males and 38 females with an average age of $6.2 \pm 3.3$ months. Ventricular and atrial defects were repaired generally with single-patch technique using autogenous pericardium.

Results: Preoperative catheterization and angiography was performed in 41 patients. Single patch and modified single patch techniques were preferred in 57 and 9 patients respectively. The average duration for respiratory support, intensive care unit stay and discharge from hospital were $36 \pm 49.3$ hours, $4.1 \pm 1.9$ days, and $10.1 \pm 3.3$ days respectively. In the left atrioventricular valve mild, moderate and severe regurgitation were detected in $44(66.6 \%), 17(25.7 \%)$ and 2 (3\%) patients postoperatively. No regurgitation was determined in 3 patients $(4.5 \%)$. Two cases ended up with mortality (3\%).

Conclusion: Single patch repair technique can provide satisfactory surgical outcomes in patients with complete atrioventricular septal defect.
More Information

*Address for Correspondence: Dr. Can Vuran, Assistant Professor, University of Health Sciences, Istanbul, Umraniye Training and research Hospital, Department of Pediatric Cardiovascular Surgery, Elmalıkent, Adem Yavuz Cd.No:1, 34764 Ümraniye Istanbul, Turkey, Tel: +90-216- 6507676; +90-5324312831; Email: canvuran@hotmail.com

Submitted: 06 March 2020

Approved: 30 April 2020

Published: 02 May 2020

How to cite this article: Vuran $C$, Yoruker $U$, Omay O, Saritas B, Ayabakan C, et al. Our experience with single patch repair of complete atrioventricular septal defects. J Cardiol Cardiovasc Med. 2020; 5: 105-108.

DOI: 10.29328/journal.jccm.1001095

ORCiD: orcid.org/0000-0002-8790-6205

Copyright: () 2020 Vuran C, et al. This is an open access article distributed under the Creative Commons Attribution License, which permits unrestricted use, distribution, and reproduction in any medium, provided the original work is properly cited.

Keywords: Atrioventricular septal defect; Complete repair; Congenital cardiac surgery; Single patch

Check for updates

\section{Introduction}

Atrioventricular septal defects (AVSDs) occur as a result of faulty development of endocardial cushion tissues during embryonic life. This spectrum ranges from a primum atrial septal defect and cleft mitral valve (a partial AVSD), to defects involving both the primum atrial septum and inlet ventricular septum in the presence of a common atrioventricular valve (complete AVSD). These defects, particularly the complete form, are easy to detect in the fetal or neonatal period and are an important source of cardiac morbidity and mortality in this age group [1]. Treatment for the complete AVSD is primarily surgical. Operative morbidity and mortality for this procedure has dramatically improved over the past 20 years [2].

Various surgical approaches have been developed for the successful repair of complete atrioventricular septal defect (CAVSD). However, the technique used to close the interventricular communication is still debated $[1,2]$.

Our study focused on the postoperative outcomes of our 
surgical technique and evaluated the usefulness of the singlepatch technique.

\section{Patients and methods}

A total of 66 patients with CAVSD's have been operated in a tertiary care center. Informed consent was obtained for all patients. This study was approved by Institutional Review Board.

Our series consisted of 28 males and 38 females with an average age of $6.2 \pm 3.3$ months. Down syndrome was found in $45(68.1 \%)$ of patients. All operations were performed through a median sternotomy under moderate hypothermic cardiopulmonary bypass. Myocardial protection was achieved via injection of antegrade blood cardioplegia into the aortic root. Ventricular and atrial defects were repaired with singlepatch technique using autogenous pericardium treated with glutaraldehyde. In 9 patients with restrictive ventricular septal defect (VSD), modified single patch (Wilcox) technique was used. Modified single patch (Wilcox) technique was used in patients with small VSD. In all procedures, glutaraldehyde treated autogenous pericardium was used. In all cases, mitral cleft was closed with single sutures. Except for the Wilcox technique, coronary sinus was left draining to the left atrium. The hospital records, operative and follow-up notes were reviewed retrospctively in this report.

All data analyses were performed with SPSS.13 for windows statistical package. Continuous variables were expressed as median and range, means and standard deviations. Categorical data were given as percentages. Paired and unpaired Student's t tests were used as appropriate to analyse continuous data, and the $\chi^{2}$ and Fisher's exact tests were used to analyse discrete data. In all cases $\mathrm{p}$ values less than 0.05 were considered to be significant.

\section{Results}

Preoperative catheterization and angiography was performed in 41 patients, $29(70,7 \%)$ of them had Down Syndrome. The mean pulmonary vascular resistance was 2.1 $\pm 1 \mathrm{U}$. Vascular resistance was slightly higher in patients with Down syndrome but was not statistically significant $(p: 0,178)$.

According to Rastelli classification, 54 cases were type A and 12 cases were type B. In the common atrioventricular (AV) valve mild, moderate and severe regurgitation were detected in 25 (37.8\%), 32 (48.4\%) and 9 (13.6\%) patients preoperatively. There was no statistically significant difference in valve failure and mortality in patients undergoing preoperative angiography $(p: 0,213)$.

Seven patients (10.6\%) were in critical cardiac decompensation prior to operation. Eight patients (15.1\%) were entubated before surgery. Fifty-seven patients had a nonrestrictive inlet VSD, 9 patients had a restrictive VSD. Single patch and modified single patch (Wilcox) were preferred in 57 and 9 patients respectively. The average duration for respiratory support, intensive care unit stay and discharge from hospital were $36 \pm 49.3$ hours, $4.1 \pm 1.9$ days, and 10.1 \pm 3.3 days respectively. Three of our patients had a residual VSD smaller than $3 \mathrm{~mm}$ and nine had smaller than $2 \mathrm{~mm}$ in the early period. There was no difference between the techniques in terms of residual VSD. Complete heart block was not detected in any however, two patients had undergone right diaphragmatic plication due to paralysis. One patient, whose postoperative left ventricular outflow gradient increased from $10 \mathrm{mmHg}$ to $100 \mathrm{mmHg}$, was operated due to left ventricular outflow obstruction. Postoperative mild, moderate and severe left $\mathrm{AV}$ valve regurgitation were detected in 44 (66.6\%), 17 $(25.7 \%)$ and 2 (3\%) patients respectively. There was no regurgitation in 3 patients $(4,5 \%)$. Two cases ended up with mortality (3\%). Both cases were entubated before surgery. They had severe valve insufficiency after surgery and died after sepsis.

\section{Discussion}

CAVSD describes a constellation of congenital cardiac structural abnormalities characterized by an ostium primum atrial septal defect, a common $\mathrm{AV}$ valve, and a nonrestrictive defect on the ventricular septal inflow. The latter, together with the amount of $\mathrm{AV}$ valve regurgitation, determines the onset of symptoms. In our series, preoperative valve failure was not associated with mortality, whereas postoperative valve failure was associated with mortality. The pulmonary vascular disease becomes irreversible over time, therefore CAVSD should be repaired before the onset of irreversible pulmonary hypertension. This is preferably scheduled before the sixth to twelfth months of life. Complete atrioventricular septal defects are associated with high-flow systemic pressures in the pulmonary vasculature leading to fibrosis and intimal hyperplasia. This eventually reduces the total crosssectional area of the pulmonary vascular bed. Surgery within 6 months usually prevents these irreversible obliterative changes, and therefore, this pathology should be repaired as early as possible $[3,4]$. In our series, angiography was performed in 28 patients over 6 months of age, 8 patients with severe heart failure and 5 patients for anatomical evaluation. No irreversible pulmonary hypertensive vascular bed was observed in any patient and there was no association between age groups and irreversible pulmonary hypertension in our series.

Palliation with pulmonary artery banding is now seldom indicated and has now been abandoned for a single-stage definitive surgical repair. In recent years, the surgical anatomy of CAVSD has been understood better, as well as improvements in surgical techniques and postoperative care. This in turn has led to a decrease in the mortality rate among this patient population and it is accepted that repair at an early age was beneficial $[5,6]$. There are no patients who underwent a pulmonary banding operation before the correction attempt in our series. 
In fact, early correction could partially eliminate the incidence of left $\mathrm{AV}$ valve regurgitation in the postoperative period, which remains the most important factor of postoperative morbidity and mortality. Other risk factors for mortality after surgical repair include the patient's age at the time of surgery, the severity of preoperative common $\mathrm{AV}$ valve regurgitation, the presence of associated cardiac anomalies, and the degree of functional disability [3-5]. In our series two patients ended up with mortality. Although their preoperative valve regurgitation were mild to moderate, they had moderate and severe regurgitation following CAVSD repair. In our series, preoperative valve failure was not associated with mortality, whereas postoperative valve failure was associated with mortality. No difference was observed in patients undergoing early or late surgery in terms of valve failure. Although it was not statistically significant, it was observed that postoperative valve functions were better in patients with small primum atrial septal defect. This suggests that the anulus is more stable in patients with small atrial septal defect.

The main difference between the various techniques proposed for the correction of atrioventricular septal defect is in using either a single or double patch technique in closing the septal defects. Both these techniques seem to be equally efficacious [6,7]. In our data, it is compatible with the literature and there was no difference between the techniques except residual VSD size. As always, the surgeon's experience with a particular technique and the ability to adapt to the highly variable pathologic abnormality of the CAVSD is probably more important than just the technique itself. It would, however, be desirable to adopt a simpler and reproducible technique that, with some customization, can be used consistently from one patient to another. Moreover, improvements in anesthetic and intensive care have made it a relatively safe procedure. These patients are also being referred at a younger age than previously and, consequently, before the onset of unmanageable pulmonary hypertension. It is well known that the vascular disease may develop faster in children with Down's syndrome [4].

Many surgical techniques have been proposed for the repair of these defects, but a more customized approach may be more appropriate given their highly variable morphology. The strategy of repair using the one- or two-patch technique and routine left AV valve closure is associated with low early mortality for patients with isolated defects, though reoperation for left $\mathrm{AV}$ valve regurgitation remains a source of late major morbidity $[8,9]$.

The primary repair is feasible even in very small infants. 38 of our patients were younger than six months and 14 of them were younger than 4 months of age. No significant difference was observed between the groups. A faulty indication for surgery such as the preoperative underestimation of the degree of the underdevelopment of the left ventricle may be the actual cause for mortality [3-5]. Evaluation of left ventricular injection in angiography is important for this problem. This problem was not encountered after careful evaluation of angiographic and echocardiographic data in our patients.

Excellent short- and mid-term results of the modified single-patch technique have been reported. The expected advantage of the single-patch technique is the simplicity. However, concern exists that any left AV valve deformity, or residual VSD would deteriorate after repair with the modified single-patch technique [6]. 12 of our patients had a residual VSD smaller than $3 \mathrm{~mm}$ in the early period and there was no difference between the techniques in terms of residual VSD.

Our study has several limitations: it was a non-randomized design, with a relatively small study group and a short follow-up period. In addition, the operations were performed by several surgeons who used slightly different surgical techniques. Consequently, a larger group of patients, longer follow-up, and randomized studies are required to demonstrate that the single-patch technique is indeed an effective procedure.

The single-patch technique is a simple and practical technique that is easily applicable in complete AVSD with low rates of mortality and morbidity. The wide application of this technique and ways to avoid complications remain a challenging problem.

\section{Conclusion}

In CAVSD, early surgical intervention is the favorable treatment method despite the complexity of the lesion. In the current era, early repair of CAVSD using the single patch repair technique seems safe and effective.

\section{References}

1. Redmond JM, Silove ED, De Giovanni JV, Wright JG, Sreeram N, et al. Complete atrioventricular septal defects: the influence of associated cardiac anomalies on surgical management and outcome. Eur $\mathrm{J}$ Cardiothorac Surg. 1996; 10: 991-995.

PubMed: https://www.ncbi.nlm.nih.gov/pubmed/8971512

2. Kobayashi M, Takahashi $\mathrm{Y}$, Ando M. Ideal timing of surgical repair of isolated complete atrioventricular septal defect. Interact Cardiovasc Thorac Surg.2007; 6: 24-26.

PubMed: https://www.ncbi.nlm.nih.gov/pubmed/17669760

3. Xie O, Brizard CP, d'Udekem Y, Galati JC, Kelly A, et al. Outcomes of repair of complete atrioventricular septal defect in the current era. Eur J Cardiothorac Surg. 2014; 45: 610-617.

PubMed: https://www.ncbi.nlm.nih.gov/pubmed/24057432

4. Tumanyan MR, Filaretova OV, Chechneva VV, Gulasaryan RS, Butrim IV, et al. Repair of complete atrioventricular septal defect in infants with down syndrome: outcomes and long-term results. Pediatr Cardiol. 2015; 36: 71-75.

PubMed: https://www.ncbi.nlm.nih.gov/pubmed/25099029

5. Frontera-Izquierdo P, Cabezuelo-Huerta G. Natural and modified history of complete atrioventricular septal defect--a 17 year study. Arch Dis Child. 1990; 65: 964-967.

PubMed: https://www.ncbi.nlm.nih.gov/pubmed/2221969 
6. Jeong IS, Lee CH, Lee C, Lim HG, Kim IS, et al. Surgical outcomes of the modified single-patch technique in complete atrioventricular septal defect. Interact Cardiovasc Thorac Surg. 2009; 8: 435-438.

PubMed: https://www.ncbi.nlm.nih.gov/pubmed/19181697

7. Okada Y, Tatsuno K, Kikuchi T, Takahashi Y, Shimokawa T. Complete atrioventricular septal defect associated with tetralogy of fallot: surgical indications and results. Jpn Circ J. 1999; 63: 889-892.

PubMed: https://www.ncbi.nlm.nih.gov/pubmed/10598897
8. Murashita T, Hatta E, Kubota T, Imamura M, Shiiya N, et al. Simplified technique for patch augmentation and chorda reconstruction of left atrioventricular valve in complete atrioventricular septal defect. J Card Surg. 2003; 18: 253-256.

PubMed: https://www.ncbi.nlm.nih.gov/pubmed/12809400

9. Gatzoulis MA, Shore D, Yacoub M, Shinebourne EA. Complete atrioventricular septal defect with tetralogy of Fallot: diagnosis and management. Br Heart J. 1994; 71: 579-583.

PubMed: https://www.ncbi.nlm.nih.gov/pmc/articles/PMC1025458/ 